\title{
Hybrid Zero Dynamics of Planar Bipedal Walking
}

\author{
J.W. Grizzle ${ }^{1}$ and E.R. Westervelt ${ }^{2}$ \\ 1 Control Systems Laboratory, Electrical Engineering and Computer Science \\ Department, University of Michigan, 1301 Beal Avenue, Ann Arbor, MI \\ 48109-2122 USA, grizzle@umich.edu \\ 2 Department of Mechanical Engineering, The Ohio State University, 201 West \\ 19th Avenue, Columbus, OH 43210-1142 USA, westervelt.4@osu.edu
}

Summary. Models of bipedal robots in motion are fundamentally hybrid due to the presence of continuous phases, discrete transitions, and unilateral constraints arising from the contact forces between the robot and the ground. A major challenge in the control of bipedal robots has been to create a feedback theory that provides systematic synthesis methods, provable correctness and computational tools for designing asymptotically stable, periodic walking motions, especially walking motions that are dynamic unlike the quasi-static, flat-footed gaits that are prevalent in today's machines. This chapter highlights the fundamental role of zero dynamics in obtaining truly dynamic walking gaits that include underactuated phases. The theoretical analysis is verified with experimental work.

\section{Introduction}

Feedback control is an integral part of any biped, whether biological or mechanical. With the exception of "passive" robots that exhibit a very limited range of stable walking on an inclined plane, without any sensing and control [5], bipeds are dynamically unstable. Said another way, without a properly functioning control system, a biped stumbles and falls.

Models of bipedal robots are quite complex. They are hybrid, nonlinear, and typically, high dimensional. In addition, as will be explained later, the continuous portion of the dynamics is effectively underactuated. A further complication is that a steady walking cycle is a non-trivial periodic motion. This means that standard stability tools for static equilibria do not apply. Instead, one must use tools appropriate for the study of periodic orbits, such as Poincaré return maps. It is of course well known how to use numerical methods to compute a Poincaré return map and to find fixed points of it [12]. The drawback in such a direct approach is that it does not yield sufficient insight for feedback design and synthesis. An extension of the notion of the zero dynamics to the hybrid models arising in bipedal locomotion leads to a 
feedback design process in which Poincaré stability analysis can be directly and insightfully incorporated into feedback synthesis.

Early definitions of the zero dynamics of a time-invariant nonlinear control system were proposed by Krener and Isidori in 1980 (using controlledinvariant distributions), by Byrnes and Isidori in 1984, and Marino in 1985 (using inverse systems) as a tool for feedback design and stability analysis. An important refinement of the concept was achieved by Isidori and Moog in 1988 [10], where three equivalent state-space characterizations of the zero dynamics of a linear time-invariant system were evaluated and compared for nonlinear systems, including the now-well-known definition of the zero dynamics as the restriction dynamics to the largest controlled-invariant manifold contained in the zero set of the output. Which of the definitions to adopt in nonlinear control was not settled until the hugely-influential 1991 paper [3] by Byrnes and Isidori that treated stabilization of equilibrium points on the basis of the zero dynamics. The notion of a hybrid zero dynamics builds on this fundamental work.

For the hybrid closed-loop system consisting of a biped robot, its environment, and a given feedback controller, the objective during the analysis phase is to be able to determine if periodic orbits exist and, if they exist, whether they are asymptotically stable. In the ensuing feedback synthesis phase, the objective is to optimize over a class of stabilizing feedback controllers in order to achieve performance objectives, such as minimal peak actuator torques and walking with a given average speed.

\section{Why Study Underactuation?}

An important source of complexity in a bipedal robot is the degree of actuation of the model, or more precisely, the degree of underactuation. It is assumed here that the robot's legs are terminated in points, and consequently, no actuation is possible at the end of the stance leg. It follows that the mechanical model is underactuated during single support, as opposed to fully actuated (a control at each joint and at the contact point with the ground). One could be concerned that "real robots have feet", and thus, while the analysis of pointfeet models may be of interest mathematically, it is "misguided for practical robotics". Focusing on underactuation is important for at least two reasons.

If one takes human walking as the defacto standard against which mechanical bipedal walking is to be compared, then the flat-footed walking achieved by current robots needs to be improved. In particular, toe roll toward the end of the single support phase needs to be allowed as part of the gait design. Currently, this is not allowed specifically because it leads to underactuation, ${ }^{3}$ which cannot be treated with a control design philosophy based on trajectory

\footnotetext{
${ }^{3}$ When the foot is rotating about the toe, one effectively has a point contact with no actuation.
} 
tracking and the quasi-static stability criterion, known as the Zero Moment Point (ZMP) [14], as is currently practiced widely in the bipedal robotics community.

\section{Hybrid Model of a Bipedal Walker}

This section introduces a hybrid dynamic model for walking motions of a planar bipedal robot with point feet. The robot is assumed to consist of $N \geq 2$ rigid links with mass connected via rigid, frictionless revolute joints to form a single open kinematic chain lying in a plane. It is further assumed that there are two identical sub-chains called the legs, connected at a common point called the hip, and, optionally, additional sub-chains that may be identified as a torso, arms, a tail, etc. Since each leg end is terminated in a point, either the robot does not have feet, or it is walking tip-toe. A typical allowed robot is depicted in Fig. 1(a), which is intentionally suggestive of a human form. All motions will be assumed to take place in the sagittal plane and consist of successive phases of single support (stance leg on the ground and swing leg in the air) and double support (both legs on the ground). Conditions that guarantee the leg ends alternate in ground contact-while other links such as the torso or arms remain free-will be imposed during control design. A rigid impact is used to model the contact of the swing leg with the ground. Further details on the model are given in [17, Sec. II], along with assumptions on the walking gait (symmetric, motion from left to right, instantaneous double support phase, no slipping or rebound at impact).

The distinct phases of walking naturally lead to mathematical models that are comprised of two parts: the differential equations describing the dynamics during the swing phase and a model that describes the dynamics when a leg end impacts the ground. For the models developed here, the ground-also called a walking surface - is assumed to be smooth and perpendicular to the gravitational field, that is, the ground is assumed to be flat as opposed to sloped or terraced.

\subsection{Lagrangian Swing Phase Model}

The swing phase model corresponds to a pinned open kinematic chain. It is assumed that only symmetric gaits are of interest, and hence it does not matter which leg end is pinned. The swapping of the roles of the legs will be accounted for in the impact model.

Let $\mathcal{Q}$ be the $N$-dimensional configuration manifold of the robot when the stance leg end is acting as a pivot and let $q:=\left(q_{1} ; \cdots ; q_{N}\right) \in \mathcal{Q}$ be a set of generalized coordinates and denote the potential and kinetic energies by $V(q)$ and $K(q, \dot{q})=\frac{1}{2} \dot{q}^{\prime} D(q) \dot{q}$, respectively, where the inertial matrix $D$ is

positive definite on $\mathcal{Q}$. The dynamic model is easily obtained with the method of Lagrange, yielding the mechanical model 


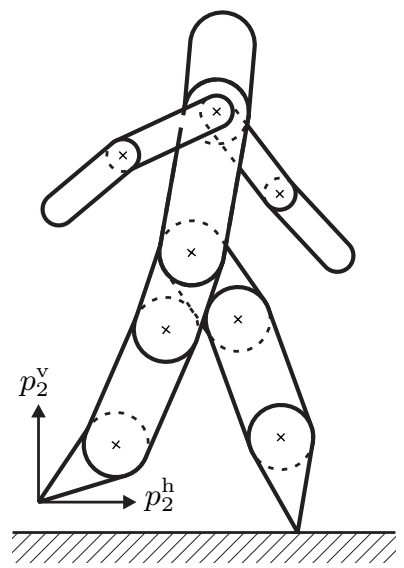

(a)

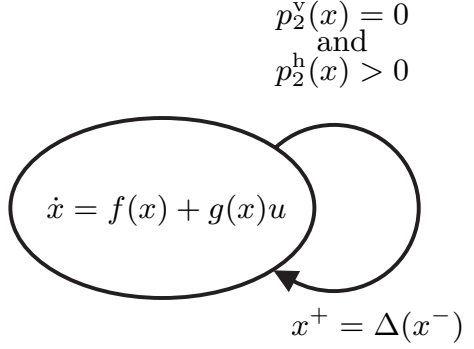

(b)

Fig. 1. (a) A typical planar robot model analyzed here. For later use, Cartesian coordinates are indicated at the swing leg end. (b) Hybrid model of walking with point feet. Key elements are the continuous dynamics of the single support phase, written in state space form as $\dot{x}=g(x)+g(x) u$, the switching or impact condition, $p_{2}^{\mathrm{v}}(q)=0$ and $p_{2}^{\mathrm{h}}(q)>0$, which detects when the height of the swing leg above the walking surface is zero and the swing leg is in front of the stance leg, and the re-initialization rule coming from the impact map, $\Delta$.

$$
D(q) \ddot{q}+C(q, \dot{q}) \dot{q}+G(q)=B(q) u,
$$

where $u=\left(u_{1} ; \cdots ; u_{N-1}\right) \in \mathbb{R}^{(N-1)}$, where $u_{i}$ is the torque applied between the two links connected by joint-i, and there is no torque applied between the stance leg and ground. The model is written in state space form by defining

$$
\begin{aligned}
\dot{x} & =\left[\begin{array}{c}
\dot{q} \\
D^{-1}(q)[-C(q, \dot{q}) \dot{q}-G(q)+B(q) u]
\end{array}\right] \\
& =: f(x)+g(x) u
\end{aligned}
$$

where $x:=(q ; \dot{q})$. The state space of the model is $\mathcal{X}=T \mathcal{Q}$. For each $x \in \mathcal{X}$, $g(x)$ is a $2 N \times(N-1)$ matrix; its $i$-th column is denoted by $g_{i}$. In natural coordinates $(q ; \dot{q})$ for $T \mathcal{Q}, g$ is independent of $\dot{q}$.

\subsection{Impact Model}

The impact of the swing leg with the ground at the end of a step is represented with the rigid (i.e., perfectly inelastic) contact model of $[6,13]$. This model effectively collapses the impact phase to an instant in time. The impact forces are consequently represented by impulses, and a discontinuity or jump is allowed in the velocity component of the robot's state, with the configuration variables remaining continuous or constant during the impact. Since we are 
assuming a symmetric walking gait, we can avoid having to use two swing phase models - one for each leg playing the role of the stance leg-by relabeling the robot's coordinates at impact. The coordinates must be relabeled because the roles of the legs must be swapped. Immediately after swapping, the former swing leg is in contact with the ground and is poised to take on the role of the stance leg.

The relabeling of the generalized coordinates is given by a matrix, $R$, acting on $q$ with the property that $R R=I$, i.e., $R$ is a circular matrix. The result of the impact and the relabeling of the states provides an expression

$$
x^{+}=\Delta\left(x^{-}\right)
$$

where $x^{+}:=\left(q^{+} ; \dot{q}^{+}\right)\left(\right.$resp. $\left.x^{-}:=\left(q^{-} ; \dot{q}^{-}\right)\right)$is the state value just after (resp. just before) impact and

$$
\Delta\left(x^{-}\right):=\left[\begin{array}{c}
\Delta_{q} q^{-} \\
\Delta_{\dot{q}}\left(q^{-}\right) \dot{q}^{-}
\end{array}\right] .
$$

The impact map is linear in the generalized velocities. Further details are given in $[7,17]$.

\subsection{Overall Hybrid Model}

A hybrid model of walking is obtained by combining the swing phase model and the impact model to form a system with impulse effects. Assume that the trajectories of the swing phase model possess finite left and right limits, and denote them by $x^{-}(t):=\lim _{\tau / t} x(\tau)$ and $x^{+}(t):=\lim _{\tau \backslash t} x(\tau)$, respectively. The model is then

$$
\Sigma:\left\{\begin{aligned}
\dot{x} & =f(x)+g(x) u, & & x^{-} \notin \mathcal{S} \\
x^{+} & =\Delta\left(x^{-}\right), & & x^{-} \in \mathcal{S},
\end{aligned}\right.
$$

where the switching set is chosen to be

$$
\mathcal{S}:=\left\{(q, \dot{q}) \in T \mathcal{Q} \mid p_{2}^{\mathrm{v}}(q)=0, p_{2}^{\mathrm{h}}(q)>0\right\} .
$$

In words, a trajectory of the hybrid model is specified by the swing phase model until an impact occurs. An impact occurs when the state "attains" the set $\mathcal{S}$, which represents the walking surface. At this point, the impact of the swing leg with the walking surface results in a very rapid change in the velocity components of the state vector. The impulse model of the impact compresses the impact event into an instantaneous moment in time, resulting in a discontinuity in the velocities. The ultimate result of the impact model is a new initial condition from which the swing phase model evolves until the next impact. In order for the state not to be obliged to take on two values at the "impact time", the impact event is, roughly speaking, described in 
terms of the values of the state "just prior to impact" at time " $t$ " "and "just after impact" at time " $t$ ". These values are represented by the left and right limits, $x^{-}$and $x^{+}$, respectively. Solutions are taken to be right continuous and must have finite left and right limits at each impact event. Figure 1(b) gives a graphical representation of this discrete-event system.

A step of the robot is a solution of (6) that starts with the robot in double support, ends in double support with the configurations of the legs swapped, and contains only one impact event. Walking is a sequence of steps.

\section{Feedback Design via Posture Control}

Any attempt to describe walking, even something as simple as the difference between human-like walking (knees bent forward) and bird-like walking (knees bent backward), inevitably leads to a description of the posture or shape of the robot throughout a step. In other words, a description of walking involves at least a partial specification of the path followed in the configuration space of the biped; see Fig. 2. The following is one possible way to express this mathematically: let $q_{\mathrm{b}}=\left(q_{1} ; \cdots ; q_{N-1}\right)$ be a set of body coordinates for the robot and let $\theta$ be the angle of some point of the robot with respect to an inertial frame, and assume moreover that $\theta$ has been chosen so that it is strictly monotonic throughout the step. Then the path of the robot in the configuration space can be expressed as

$$
0=q_{\mathrm{b}}-h_{d}(\theta) .
$$

A natural objective is therefore: determine a feedback controller that drives asymptotically to zero the output function

$$
y=h(q):=q_{\mathrm{b}}-h_{d}(\theta) .
$$

This leads to two questions:

1. An analysis question: when will a given choice of $h_{d}(\theta)$ lead to an asymptotically stable periodic orbit (i.e., a stable walking motion)?

2. A synthesis question: how to make a choice of $h_{d}(\theta)$ that will yield an asymptotically stable periodic orbit meeting physically motivated requirements such as: energy efficiency; the robot walks at a desired speed; and the reaction forces at the leg end are such that the contact between the stance and the ground behaves as a pivot?

Addressing the first question leads to the notion of the hybrid zero dynamics, the focus of this chapter. A finite parametrization of possible paths $h_{d}(\theta, \alpha)$ via Bézier polynomials and the use of parameter optimization have been employed to address the second question. 

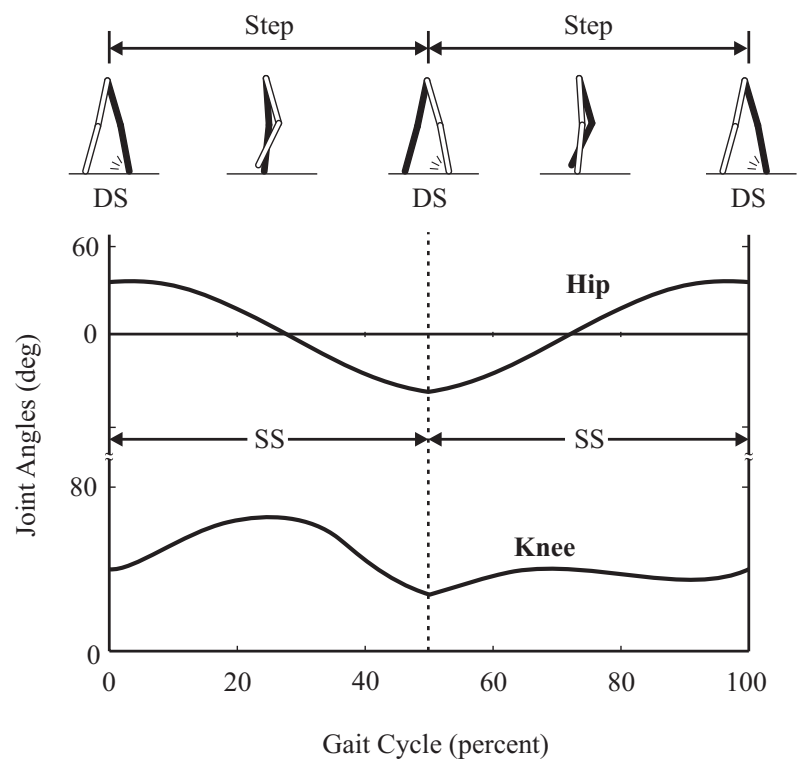

Fig. 2. Joint angles for a four-link walker over a complete gait cycle, that is, two steps. The gait cycle consists of two phases each of double support (DS) and single support (SS). Depicted are the relative hip angle and the knee angle of the leg drawn in white. The first single support phase can be thought of as a graph of (8) for the relative angles of the hip and knee during the swing phase, and the second single support phase is a graph of (8) for the relative angles of the hip and knee during the stance phase. The angle $\theta$ in (8) can be taken as the angle of the hip with respect to the ground contact point of the stance leg.

\section{The Zero Dynamics of Walking}

The zero dynamics of the hybrid model (6) with output (9) are developed in a two-step process. First, the zero dynamics of the (non-hybrid) nonlinear model consisting of the swing phase dynamics (3) and the output (9) are characterized, and then, second, an impact invariance condition is imposed on the swing-phase zero dynamics manifold in order to obtain the hybrid zero dynamics. For general hybrid systems and output functions, this approach to forming a hybrid zero dynamics is less general than directly applying the output zeroing definition of Isidori et al. [3, 9, 10]. From results in [17, 18], however, it can be deduced that for an $N$-degree of freedom biped model with one degree of underactuation and an $N-1$ dimensional output vector of the form (9), if there exists at least one periodic solution of the hybrid model (6) that zeros the output and is transversal to $\mathcal{S}$, then the approach followed here and the definition used in [9] are equivalent. 


\subsection{The Swing Phase Zero Dynamics}

The objective is to characterize the zero dynamics manifold and associated restriction dynamics for the swing-phase model (3) and output (9). The zero dynamics, by definition, is independent of the choice of coordinates and the application of regular state variable feedback [9, pp. 228]. Express the mechanical model $(1)$ in the generalized coordinates $q=\left(q_{\mathrm{b}} ; \theta\right)$. It is proven in [8, pp. 562] that the model (3) is globally feedback equivalent to

$$
\begin{aligned}
\ddot{q}_{\mathrm{b}} & =v \\
\dot{\theta} & =\frac{\bar{\sigma}_{N}}{d_{N, N}\left(q_{\mathrm{b}}\right)}-J^{\mathrm{norm}}\left(q_{\mathrm{b}}\right) \dot{q}_{\mathrm{b}} \\
\dot{\bar{\sigma}}_{N} & =-\frac{\partial V}{\partial \theta}\left(q_{\mathrm{b}}, \theta\right),
\end{aligned}
$$

where

$$
J^{\mathrm{norm}}\left(q_{\mathrm{b}}\right)=\frac{1}{d_{N, N}\left(q_{\mathrm{b}}\right)}\left[d_{N, 1}\left(q_{\mathrm{b}}\right), \cdots, d_{N, N-1}\left(q_{\mathrm{b}}\right)\right],
$$

$d_{j, k}$ is the $j$ - $k$-element of $D, \bar{\sigma}_{N}$ is the generalized momentum conjugate to $q_{N}=\theta$, and $v$ is the new input coming from the feedback transformation. Taking $\tilde{x}:=\left(q_{\mathrm{b}} ; \theta ; \dot{q}_{\mathrm{b}} ; \bar{\sigma}_{N}\right)$, the swing-phase model after feedback is expressed in state variable form as

$$
\dot{\tilde{x}}=\left[\begin{array}{c}
\dot{q}_{\mathrm{b}} \\
\frac{\bar{\sigma}_{N}}{d_{N, N}\left(q_{\mathrm{b}}\right)}-J^{\text {norm }}\left(q_{\mathrm{b}}\right) \dot{q}_{\mathrm{b}} \\
v \\
-\frac{\partial V}{\partial \theta}\left(q_{\mathrm{b}}, \theta\right)
\end{array}\right]=: \tilde{f}(\tilde{x})+\tilde{g}(\tilde{x}) v .
$$

\section{Decoupling Matrix}

Differentiating (9) twice along the dynamics (12) gives

$$
\ddot{y}=L_{\tilde{f}}^{2} h(\tilde{x})+L_{\tilde{g}} L_{\tilde{f}} h(q) v .
$$

A simple calculation gives that the decoupling matrix is

$$
L_{\tilde{g}} L_{\tilde{f}} h(\tilde{q})=\mathrm{I}_{(N-1) \times(N-1)}+\underbrace{\frac{\partial h_{d}(\theta)}{\partial \theta}}_{(N-1) \times 1} \underbrace{J^{\mathrm{norm}}\left(q_{\mathrm{b}}\right)}_{1 \times(N-1)} .
$$

It follows that [?]

$$
\operatorname{det}\left(L_{\tilde{g}} L_{\tilde{f}} h\right)(q)=1+J^{\mathrm{norm}}\left(q_{\mathrm{b}}\right) \frac{\partial h_{d}(\theta)}{\partial \theta}
$$

and is nonzero if, and only if, 


$$
d_{N, N}\left(q_{\mathrm{b}}\right)+\left[d_{N, 1}\left(q_{\mathrm{b}}\right), \cdots, d_{N,(N-1)}\left(q_{\mathrm{b}}\right)\right] \frac{\partial h_{d}(\theta)}{\partial \theta} \neq 0 .
$$

Moreover, on the open set $T \tilde{\mathcal{Q}} \subset T \mathcal{Q}$ where the determinant of the decoupling matrix is nonzero, the inverse of the decoupling matrix is

$$
\left[L_{\tilde{g}} L_{\tilde{f}} h(q)\right]^{-1}=\mathrm{I}_{(N-1) \times(N-1)}+\frac{1}{\operatorname{det}\left(L_{\tilde{g}} L_{\tilde{f}} h\right)(q)} \frac{\partial h_{d}(\theta)}{\partial \theta} J^{\mathrm{norm}}\left(q_{\mathrm{b}}\right) .
$$

The swing phase zero dynamics manifold is then ${ }^{4}$

$$
\mathcal{Z}:=\left\{x \in T \tilde{\mathcal{Q}} \mid h(x)=0, L_{f} h(x)=0\right\} ;
$$

it is a smooth two-dimensional embedded submanifold of $T \mathcal{Q}$. The feedback control

$$
\tilde{u}^{*}(\tilde{x})=-\left[L_{\tilde{g}} L_{\tilde{f}} h(q)\right]^{-1} L_{\tilde{f}}^{2} h(\tilde{x})
$$

renders $\mathcal{Z}$ invariant under the closed-loop vector field $\tilde{f}+\tilde{g} \tilde{u}^{*}$. The zero dynamics vector field is the restriction

$$
f_{\text {zero }}:=\tilde{f}+\left.\tilde{g} \tilde{u}^{*}\right|_{\mathcal{Z}} .
$$

The zero dynamics is given by

$$
\dot{z}=f_{\text {zero }}(z),
$$

for $z \in \mathcal{Z}$.

\section{Computing Terms in the Zero Dynamics}

In the coordinates $\left(q_{\mathrm{b}} ; \theta ; \dot{q}_{\mathrm{b}} ; \dot{\theta}\right)$, the zero dynamics manifold can be written as

$$
\mathcal{Z}=\left\{\left(q_{\mathrm{b}} ; \theta ; \dot{q}_{\mathrm{b}} ; \dot{\theta}\right) \mid q_{\mathrm{b}}=h_{d}(\theta), \dot{q}_{\mathrm{b}}=\frac{\partial h_{d}(\theta)}{\partial \theta} \dot{\theta}\right\} .
$$

On $\mathcal{Z}$, the generalized momentum conjugate to $\theta$ becomes

$$
\bar{\sigma}_{N}=I(\theta) \dot{\theta},
$$

where the virtual inertia $I(\theta)$ is given by

$$
I(\theta):=\left.\left[d_{N, N}\left(q_{\mathrm{b}}\right)+\left[d_{N, 1}\left(q_{\mathrm{b}}\right), \cdots, d_{N,(N-1)}\left(q_{\mathrm{b}}\right)\right] \frac{\partial h_{d}(\theta)}{\partial \theta}\right]\right|_{q_{\mathrm{b}}=h_{d}(\theta)} .
$$

On $\mathcal{Z}$, the invertibility of the decoupling matrix establishes a bijective relationship between $\bar{\sigma}_{N}$ and $\dot{\theta}$,

\footnotetext{
${ }^{4}$ By [9, pp. 230], $L_{\tilde{f}} h=L_{f} h$; moreover, (12) and (3) have the same zero dynamics because they are related by a regular state variable feedback [9].
} 


$$
\dot{\theta}=\frac{\bar{\sigma}_{N}}{I(\theta)}
$$

Restricting (10c) to $\mathcal{Z}$

$$
\dot{\bar{\sigma}}_{N}=-\left.\frac{\partial V}{\partial \theta}\left(q_{\mathrm{b}}, \theta\right)\right|_{q_{\mathrm{b}}=h_{d}(\theta)} .
$$

Defining $\xi_{1}:=\theta$ and $\xi_{2}:=\bar{\sigma}_{N}$, it follows that the swing phase zero dynamics is

$$
\begin{aligned}
& \dot{\xi}_{1}=\kappa_{1}\left(\xi_{1}\right) \xi_{2} \\
& \dot{\xi}_{2}=\kappa_{2}\left(\xi_{1}\right)
\end{aligned}
$$

where

$$
\begin{aligned}
\kappa_{1}\left(\xi_{1}\right) & =\frac{1}{I\left(\xi_{1}\right)} \\
\kappa_{2}\left(\xi_{1}\right) & =-\left.\frac{\partial V}{\partial \theta}\right|_{q_{\mathrm{b}}=h_{d}(\theta), \theta=\xi_{1}} .
\end{aligned}
$$

It is emphasized that these terms can be determined directly from the Lagrangian of the swing-phase model and the term $h_{d}$ of the output (9). In particular, there is no need to invert the inertia matrix, as would be required if the zero dynamics were computed directly from (3).

\subsection{The Hybrid Zero Dynamics}

To obtain the hybrid zero dynamics, the zero dynamics manifold must be invariant under the impact map, that is

$$
\Delta(\mathcal{S} \cap \mathcal{Z}) \subset \mathcal{Z}
$$

If $\mathcal{S} \cap \mathcal{Z}$ is nonempty, then, due to the form of the output (9), $\mathcal{S} \cap \mathcal{Z}$ is a smooth one-dimensional embedded submanifold of $T \tilde{\mathcal{Q}}$ if, and only, if $p_{2}^{\mathrm{v}}\left(h_{d}(\theta), \theta\right)$ has constant rank on its zero set. Furthermore, when the decoupling matrix is invertible, the following statements are equivalent [17]:

(a) (29) holds;

(b) $\left.h \circ \Delta\right|_{(\mathcal{S} \cap \mathcal{Z})}=0$ and $\left.L_{f} h \circ \Delta\right|_{(\mathcal{S} \cap \mathcal{Z})}=0$; and

(c) there exists at least one point $\left(q_{0}^{-} ; \dot{q}_{0}^{-}\right) \in \mathcal{S} \cap \mathcal{Z}$ such that $\bar{\sigma}_{N} \neq 0, h \circ$ $\Delta_{q}\left(q_{0}^{-}\right)=0$, and $L_{f} h \circ \Delta\left(q_{0}^{-}, \dot{q}_{0}^{-}\right)=0$.

Definition 1 (Hybrid zero dynamics [17]). Consider the hybrid model (6) and output (9). Suppose that the decoupling matrix (14) is invertible and let $\mathcal{Z}$ and $\dot{z}=f_{\text {zero }}(z)$ be the associated zero dynamics manifold and zero dynamics of the swing phase model. Suppose that $\mathcal{S} \cap \mathcal{Z}$ is a smooth, one-dimensional, 


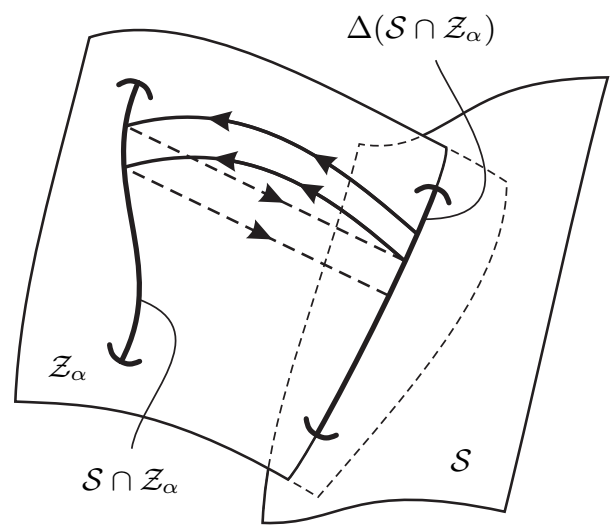

(a)

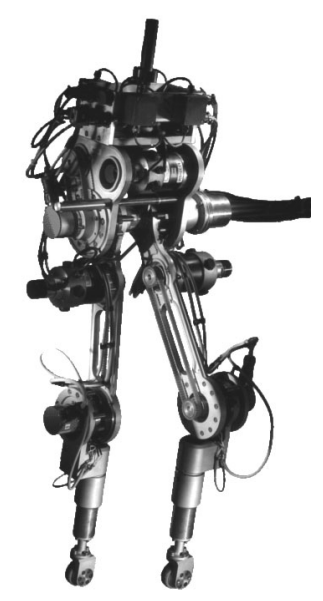

(b)

Fig. 3. In (a) geometry of the closed-loop system. In (b), the prototype RABBIT, which was developed as a French National Project by the CNRS [4]; the robot is housed in LAG, the Automatic Control Laboratory of Grenoble.

embedded submanifold of $T \mathcal{Q}$. Suppose further that $\Delta(\mathcal{S} \cap \mathcal{Z}) \subset \mathcal{Z}$. Then the nonlinear system with impulse effects,

$$
\Sigma_{\text {zero }}:\left\{\begin{array}{rlrl}
\dot{z} & =f_{\text {zero }}(z), & & z^{-} \notin \mathcal{S} \cap \mathcal{Z} \\
z^{+}=\Delta\left(z^{-}\right), & & z^{-} \in \mathcal{S} \cap \mathcal{Z},
\end{array}\right.
$$

with state manifold $\mathcal{Z}$, is the hybrid zero dynamics.

In the local coordinates $\left(\xi_{1} ; \xi_{2}\right), \mathcal{S} \cap \mathcal{Z}$ and $\Delta:\left(\xi_{1}^{-} ; \xi_{2}^{-}\right) \rightarrow\left(\xi_{1}^{+} ; \xi_{2}^{+}\right)$simplify to

$$
\begin{aligned}
\mathcal{S} \cap \mathcal{Z} & =\left\{\left(\xi_{1}^{-} ; \xi_{2}^{-}\right) \mid \xi_{1}^{-}=\theta^{-}, \xi_{2}^{-} \in \mathbb{R}\right\} \\
\xi_{1}^{+} & =\theta^{+} \\
\xi_{2}^{+} & =\delta_{\text {zero }} \xi_{2}^{-}
\end{aligned}
$$

where $\delta_{\text {zero }}$ is a constant that may be readily computed using (4) and (9) and where $\theta^{-}$and $\theta^{+}$satisfy

$$
\begin{array}{ll}
\left.p_{2}^{\mathrm{v}}\left(h_{d}\left(\theta^{-}\right), \theta^{-}\right)\right)=0, & p_{2}^{\mathrm{h}}\left(h_{d}\left(\theta^{-}\right), \theta^{-}\right)>0, \\
\left.p_{2}^{\mathrm{v}}\left(h_{d}\left(\theta^{+}\right), \theta^{+}\right)\right)=0, & p_{2}^{\mathrm{h}}\left(h_{d}\left(\theta^{+}\right), \theta^{+}\right)<0 .
\end{array}
$$

The hybrid zero dynamics is thus given by (27) during the swing phase, and at impact with $\mathcal{S} \cap \mathcal{Z}$, the re-initialization rules (31b) and (31c) are applied.

For $\theta^{+} \leq \xi_{1} \leq \theta^{-}$, define 


$$
V_{\text {zero }}\left(\xi_{1}\right):=-\int_{\theta^{+}}^{\xi_{1}} \frac{\kappa_{2}(\xi)}{\kappa_{1}(\xi)} d \xi
$$

A straightforward computation shows that $\mathcal{L}_{\text {zero }}:=K_{\text {zero }}-V_{\text {zero }}[17]$, where

$$
K_{\text {zero }}=\frac{1}{2}\left(\frac{\dot{\xi}_{1}}{\kappa_{1}\left(\xi_{1}\right)}\right)^{2},
$$

is a Lagrangian of the swing-phase zero dynamics (27). This implies, in particular, that the total energy $\mathcal{H}_{\text {zero }}:=K_{\text {zero }}+V_{\text {zero }}$ is constant along solutions of the swing-phase zero dynamics.

\subsection{Existence and Stability of Periodic Orbits}

The analysis of periodic orbits of the hybrid zero dynamics forms the basis for proposing feedback laws that induce exponentially stable walking motions in the full-dimensional hybrid model. Take the Poincaré section to be $\mathcal{S} \cap \mathcal{Z}$ and let

$$
\rho: \mathcal{S} \cap \mathcal{Z} \rightarrow \mathcal{S} \cap \mathcal{Z}
$$

denote the Poincaré (first return) map ${ }^{5}$ of the hybrid zero dynamics. Using the fact that the total energy $\mathcal{H}_{\text {zero }}$ is constant along solutions of the continuous portion of the dynamics, the Poincaré map may be shown to be

$$
\rho\left(\zeta_{2}^{-}\right)=\delta_{\text {zero }}^{2} \zeta_{2}^{-}-V_{\text {zero }}\left(\theta^{-}\right)
$$

where $\zeta_{2}^{-}:=\frac{1}{2}\left(\xi_{2}^{-}\right)^{2}$, and its domain of definition is

$$
\mathcal{D}_{\text {zero }}=\left\{\zeta_{2}^{-}>0 \mid \delta_{\text {zero }}^{2} \zeta_{2}^{-}-V_{\text {zero }}^{\max }>0\right\},
$$

where

$$
V_{\text {zero }}^{\max }:=\max _{\theta^{+} \leq \xi_{1} \leq \theta^{-}} V_{\text {zero }}\left(\xi_{1}\right) .
$$

The domain $\mathcal{D}_{\text {zero }}$ is non-empty if, and only if, $\delta_{\text {zero }}^{2}>0$. Whenever $\delta_{\text {zero }}^{2}<1$, the fixed point of (36),

$$
\zeta_{2}^{*}:=-\frac{V_{\text {zero }}\left(\theta^{-}\right)}{1-\delta_{\text {zero }}^{2}}
$$

will be exponentially stable as long as it belongs to $\mathcal{D}_{\text {zero }}$. The conditions for there to exist an exponentially stable periodic orbit of (30) are thus

$$
\begin{gathered}
\frac{\delta_{\text {zero }}^{2}}{1-\delta_{\text {zero }}^{2}} V_{\text {zero }}\left(\theta^{-}\right)+V_{\text {zero }}^{\max }<0 \\
0<\delta_{\text {zero }}^{2}<1 .
\end{gathered}
$$

Periodic orbits of the hybrid zero dynamics are periodic orbits of the fulldimensional model. Two different feedback controllers are provided in $[11,17]$ for exponentially stabilizing these orbits in the full-dimensional model, (3).

\footnotetext{
${ }^{5}$ This is in general a partial map.
} 


\section{Experimental Data}

The hybrid zero dynamics has played an integral role in the design of walking gaits for a prototype bipedal robot called RABBIT [4]; see Fig. 3(b). The analytical results of Section 5.2 are rendered useful in feedback synthesis by introducing a finite parametrization of the output (9). In particular, the function $h_{d}$ is constructed from Bézier polynomials [1], which in turn introduces free parameters $\alpha$ into the hybrid zero dynamics (30),

$$
\Sigma_{\text {zero }, \alpha}:\left\{\begin{aligned}
\dot{z} & =f_{\text {zero }, \alpha}(z), & & z^{-} \notin \mathcal{S} \cap \mathcal{Z}_{\alpha} \\
z^{+} & =\Delta\left(z^{-}\right), & & z^{-} \in \mathcal{S} \cap \mathcal{Z}_{\alpha},
\end{aligned}\right.
$$

through

$$
h_{\alpha}(q):=q_{\mathrm{b}}-h_{d}(q, \alpha) .
$$

Moreover, the hybrid invariance condition (29) can be imposed analytically; see $[17$, Thm. 4].

A minimum-energy cost criterion

$$
J(\alpha)=\frac{1}{\text { step length }} \int_{0}^{\text {step time }}\left\|u_{\alpha}^{*}(t)\right\|_{2}^{2} d t
$$

is posed, where

$$
u_{\alpha}^{*}:=-\left.\left[L_{g} L_{f} h_{\alpha}\right]^{-1} L_{f}^{2} h_{\alpha}\right|_{\mathcal{Z}}
$$

is the (unique) input to the model (3) realizing the periodic orbit of the hybrid zero dynamics. Constraints based on (40) are easily imposed to guarantee that periodic orbits exist and are exponentially stable. Additional constraints are used to prescribe walking at a desired average speed, with the (unilateral) forces on the support leg lying in the allowed friction cone. Parameter optimization is then used to minimize the cost $J(\alpha)$. Whenever a solution exists, the result is a provably stable, closed-loop system with satisfied design constraints. Typical solutions times for computing the optimal parameter value are thirty seconds to one minute in MATLAB. For further details on the procedure, see [17].

Feedback control designs based on the hybrid zero dynamics have been extensively evaluated on RABBIT. As reported in [16], natural walking motions were obtained with remarkably little trial and error. Figure 4 compares a limit cycle obtained on the robot with a limit cycle obtained with the same controller on a simulation model. For videos of RABBIT walking and running, see $[2,15]$.

\section{Conclusions}

The notion of zero dynamics has become ubiquitous in nonlinear control theory and practice. This chapter has reviewed an extension of the zero dynamics 


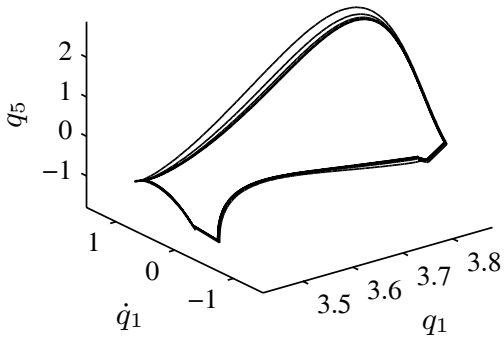

(a) simulation



(b) experiment

Fig. 4. Limit cycles of the closed-loop hybrid systems corresponding to asymptotically stable walking.

to a class of hybrid models relevant for the analysis of walking gaits in bipedal robots. The hybrid zero dynamics provides an effective tool for the analysis and synthesis of feedback controllers that induce exponentially stable, periodic walking motions in bipedal robots. Its utility has been confirmed experimentally.

\section{Acknowledgements}

The work of J.W. Grizzle and E.R. Westervelt was supported by National Science Foundation grants ECS-0600869 and CMS-0408348, respectively.

\section{References}

1. P. Bézier. Numerical Control: Mathematics and Applications. John Wiley \& Sons, New York, 1972.

2. G. Buche. ROBEA Home Page. http://robot-rabbit.lag.ensieg.inpg.fr/ English/, 2007.

3. C. Byrnes and A. Isidori. Asymptotic stabilization of nonlinear minimum phase systems. IEEE Transactions on Automatic Control, 376:1122-37, 1991.

4. C. Chevallereau, G. Abba, Y. Aoustin, F. Plestan, E. R. Westervelt, C. Canudas, and J. W. Grizzle. RABBIT: a testbed for advanced control theory. IEEE Control Systems Magazine, 23(5):57-79, October 2003.

5. S. H. Collins, A. Ruina, R. Tedrake, and M. Wisse. Efficient bipedal robots based on passive-dynamic walkers. Science, 307:1082-5, 2005.

6. B. Espiau and A. Goswani. Compass gait revisited. In Proc. of the IFAC Symposium on Robot Control, Capri, Italy, pages 839-46, September 1994.

7. J. W. Grizzle, G. Abba, and F. Plestan. Asymptotically stable walking for biped robots: Analysis via systems with impulse effects. IEEE Transactions on Automatic Control, 46:51-64, January 2001. 
8. J. W. Grizzle, C. H. Moog, and C. Chevallereau. Nonlinear control of mechanical systems with an unactuated cyclic variable. IEEE Transactions on Automatic Control, 30(5):559-76, May 2005.

9. A. Isidori. Nonlinear Control Systems. Springer-Verlag, Berlin, third edition, 1995.

10. A. Isidori and C. H. Moog. On the nonlinear equivalent of the notion of transmission zeros. In C. Byrnes and A. Kurzhanski, editors, Proc. of the IIASA Conference: Modeling and Adaptive Control, pages 146-57, Berlin, 1988. SpringerVerlag.

11. B. Morris and J. W. Grizzle. A restricted Poincaré map for determining exponentially stable periodic orbits in systems with impulse effects: Application to bipedal robots. In Proc. of the 2005 IEEE International Conference on Decision and Control European Control Conference, Seville, Spain, pages 4199-206, 2005.

12. T. S. Parker and L. O. Chua. Practical Numerical Algorithms for Chaotic Systems. Springer-Verlag, New York, 1989.

13. C.-L. Shih and W. A. Gruver. Control of a biped robot in the double-support phase. IEEE Transactions on Systems, Man and Cybernetics, 22(4):729-35, 1992.

14. M. Vukobratović, B. Borovac, D. Surla, and D. Stokic. Biped Locomotion. Springer-Verlag, Berlin, 1990.

15. E. R. Westervelt. Eric Westervelt's publications. http://www.mecheng.osu. edu/ westerve/publications/, 2007.

16. E. R. Westervelt, G. Buche, and J. W. Grizzle. Experimental validation of a framework for the design of controllers that induce stable walking in planar bipeds. International Journal of Robotics Research, 23(6):559-82, 2004.

17. E. R. Westervelt, J. W. Grizzle, and D. E. Koditschek. Hybrid zero dynamics of planar biped walkers. IEEE Transactions on Automatic Control, 48(1):42-56, January 2003.

18. E. R. Westervelt, B. Morris, and K. D. Farrell. Analysis results and tools for the control of planar bipedal gaits using hybrid zero dynamics. Autonomous Robots, 2007. In press. 